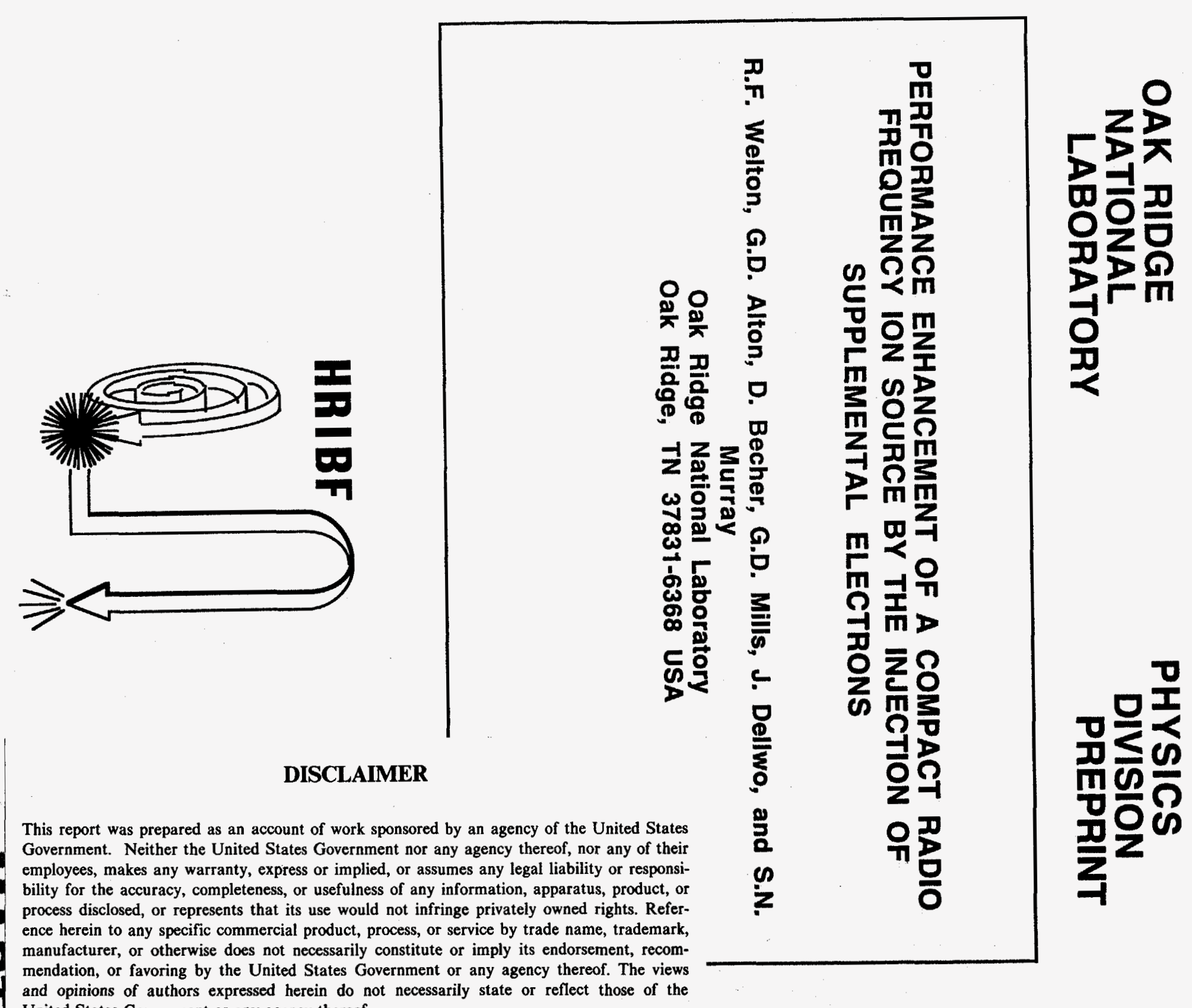




\section{DISCLAIMER}

Portions of this document may be illegible in electronic image products. Images are produced from the best available original document. 


\title{
Performance enhancement of a compact radio frequency ion source by the injection of supplemental electrons
}

\author{
R.F. Welton, G.D Alton. D. Becher, G.D. Mills, J. Dellwo and S.N. Murray \\ Oak Ridge National Laboratory*, P.0. Box 2008, Oak Ridge, Tennessee 37831
}

\begin{abstract}
A versatile. high brightness, volume type, low power $R F$ source. capable of producing positive ion beams with intensities as high as $1 \mathrm{~mA}$ from gaseous feed materiais and microamperes of negative ion beams has been characterized. The source can also be operated as a plasma sputter negative ion source to generate up to $1 \mathrm{~mA}$ of a selected species. The performance of the source in the positive and negative volume modes of operation can be greatly enhanced by addition of a removable, water cooled filament assembly in place of the negative sputter probe. For example, the material utilization efficiencies of gaseous feed species can be more than doubled, total current intensities increased up to $40 \%$, molecular dissociation fractions increased by $20 \%$ and minimum operating pressures reduced by a factor of four when operated in the volume mode. These added electrons also favorably effect, as a consequence of lower pressures, the emittance apparently through a reduction of scattering in the beam through the transport system. A brief description of the source and performance data for the positive volume mode of operation will be presented.
\end{abstract}

\section{INTRODUCTION}

The design features of the source are described in reference 1. Plasma sputter negative ion sources of this type have been utilized by several groups for a number of applications including accelerator based atomic and nuclear physics, material processing and isotope separation (see e.g. the references contained in ref. 1). In addition to the sputter mode of operation, both positive \& negative ions can be extracted directly from the $R F$ plasma when operated in the volume mode. The capability to operate in any of these three modes greatly extends the number and variety of ionic species that can be generated by the source. Because of this versatility the source can be used for numerous applications [1]. Source reliability, multimode operation, high gas load tolerance, long lifetime. low emittance and high efficiency for dissociative ionization of molecules into positive and negative singly charged atomic ions also make the radio frequency ion source a candidate for Radioactive Ion Beam (RIB) generation. Howerver issues concerning the ionization efficiencies when operated in the positive mode have not yet been resolved for these appications.

The effect of injecting electrons into a high frequency discharge ion source bas been explored extensively for microwave ECR sources [2-9] and to a lesser degree in radio frequency ion sources [10]. Lynies has shown [2] that the performance of microwave ECR sources could be enhanced through the addition of injected electrons either from a gun of by a coating of $\mathrm{SiO}_{2}$ on the metallic plasma chamber. Others employ a plasma cathode or biased disk technique to augment the electron density in the main stage of the source [8.9], Abdelaziz et al [10] has explored the improvement to beam brightness of a magnetized radial extraction RF ion source, finding nearly an order of magnitude improvement in electron density, an increase in extracted beam current and a decrease in emittance resulting in a significant increase in ion beam brightness. In this work, we characterize the performance of a compact RF source for operation in the volume mode for the generation of positive ions. Total analyzed current yields, ionization efficiency, molecular dissociation and emittance have been measured with the filament on and off.

\footnotetext{
* Managed by Martin Marietta Energy systems. Inc.. under contract No. DE-AC05-840R21400 with the U.S. Department of Energy.
}

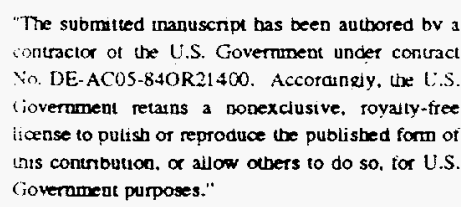




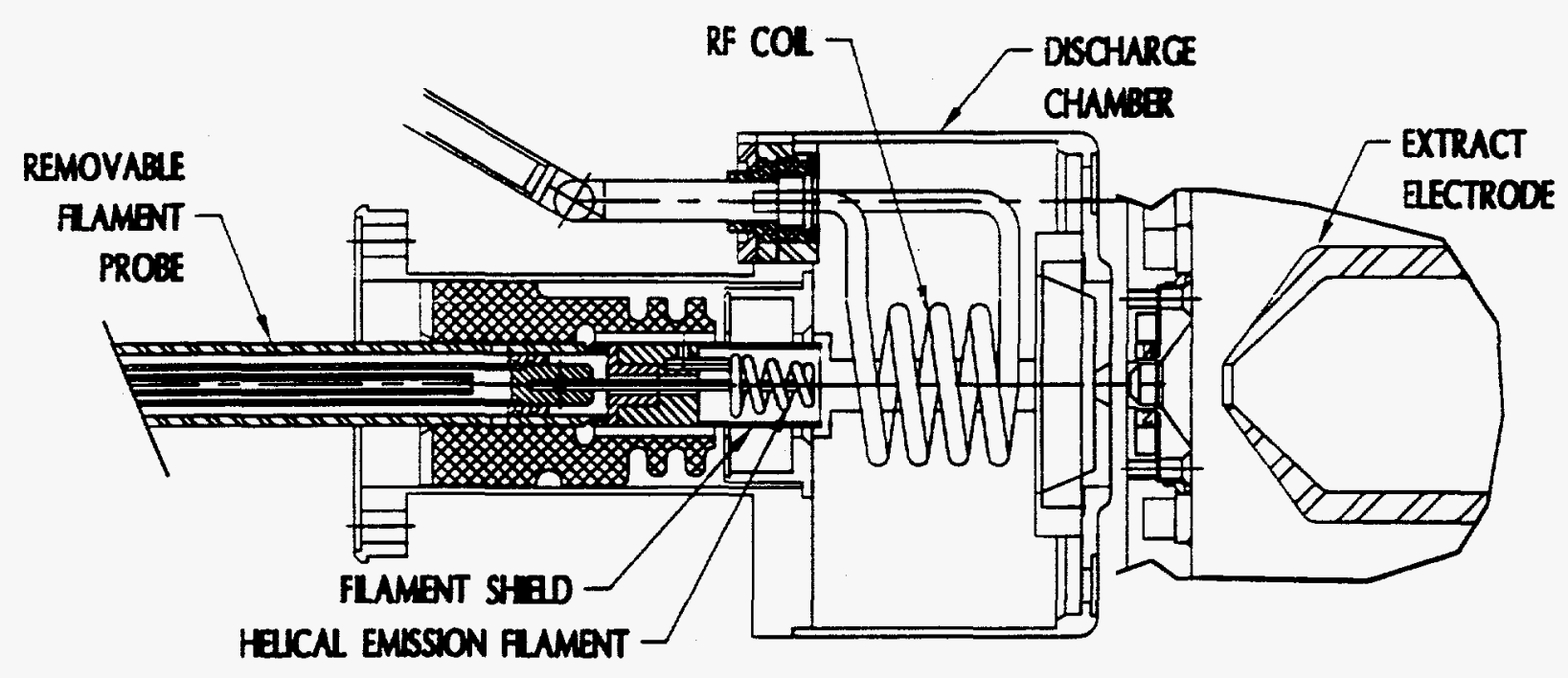

Figure 1: Schematic view of ion source showing the removable filament assembly.

\section{THE ION SOURCE AND FILAMENT PROBE ASSEMBLE}

Fig. 1 shows a cross sectional view of the RF source with the removable filament assembly in place. Radio frequency power is coupled into the plasma through a copper coil shown in the figure, matching is achieved by a network controlled remotely and located adjacent to the RF feedthrough. Power is generated in a $0-1 \mathrm{~kW}, 13.56 \mathrm{MHz}$ supply. Atomic and molecular gas mixtures can be fed into the source from a system of variable and calibrated leaks through an opening directed through the RF coil windings. A converging helical tantalum element of thickness $1.5 \mathrm{~mm}$ is raised to emission temperature by a current of $\sim 70 \mathrm{~A}$ flowing through a water cooled copper central conductor and out through a copper shield. High temperature components are isolated from the copper by a tantalum cylinder which forms a shield about the filament to prevent direct bombardment of the copper RF coil. The entire assembly can be biased with respect to the plasma.

\section{EXPERIMENTAL RESULTS}

Total extracted argon current. nitrogen dissociation fractions and trace flow $\left(9 \times 10^{.5} \mathrm{sccm}\right) \mathrm{Xe}$ efficiencies were measured with the tilament on and off as a function of operating pressure. RF power and extraction voltage. Optimal conditions of filament operation were $70 \mathrm{~A}$ heating current $10 \mathrm{~V}$ bias and an emission current of $1.5 \mathrm{~A}$. The variation of the ion current, dissociation fractions and Xe efficiencies with RF power is shown in fig. 2. In each case, the source was maintained at the lowest pressure that allowed reliable operation. Use of the filament resulted in a lowering of the normalized emittance from 2.4 to $2.12 \pi \mathrm{mm} \operatorname{mrad}(\mathrm{MeV})^{1 / 2}$ for the $90 \%$ contour of the beam. In addition, the minimum pressure for stable plasma operation was reduced on the average by a factor of four.

\section{CONCLUSION}

This work quantifies the performance enhancement that can be expected with the addition of a relatively simple filament structure to an inductively coupled RF ion source. Measurements were geared to both accelerator (injector) ion source applications where intensity is desired and ISOL (radioactive beam ) applications where ionization efficiency of trace material and molecular dissociation is most important. Under optimal conditions, the ionization efficiency of a trace flow of Xe was found to increase from $8 \%$ to $16 \%$. The electrons appear to benefit the source plasma in three important respects: (1) the minimum operating pressure is lowered four fold (to $4 \times 10^{-4}$ Torr in the source), (2) the emittance is improved and (3) the electron density is increased which subsequenty lowers the plasma potential. as observed in ref. 2 and 7 . 
Nitrogen dissociation fractions

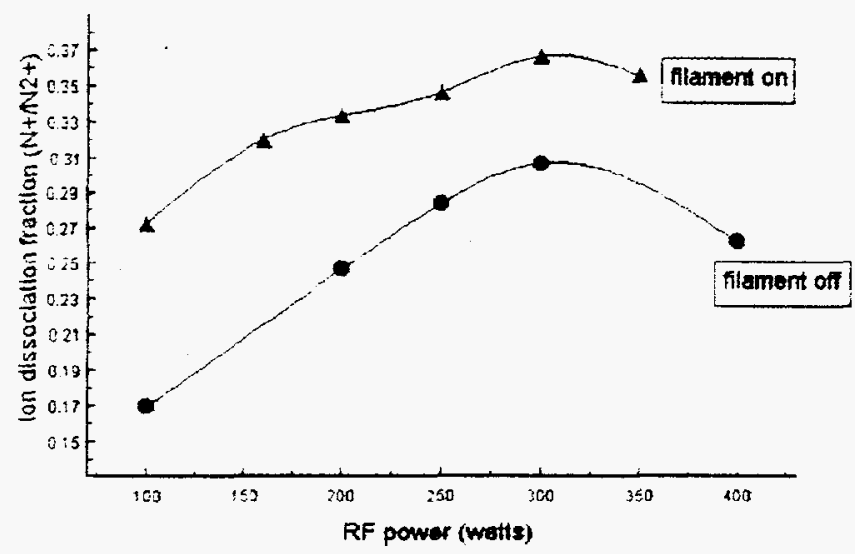

Xe ionization efficiency

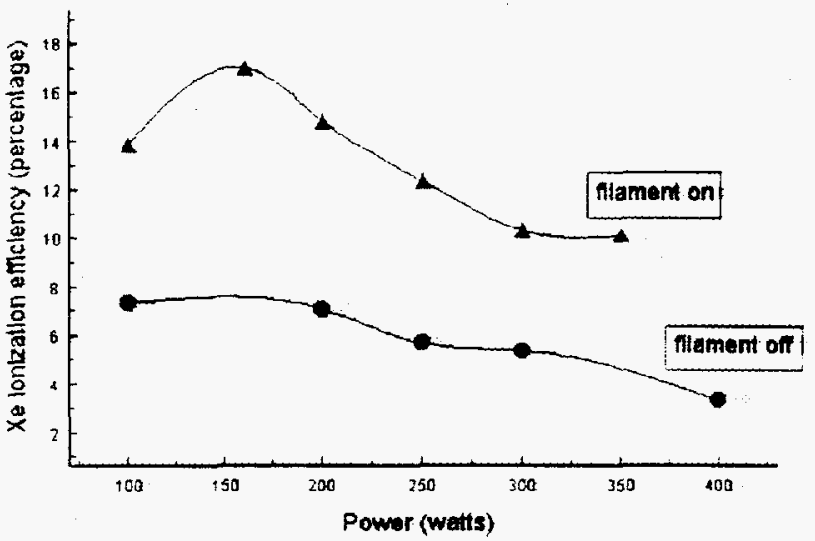

Mass analyzed argon current

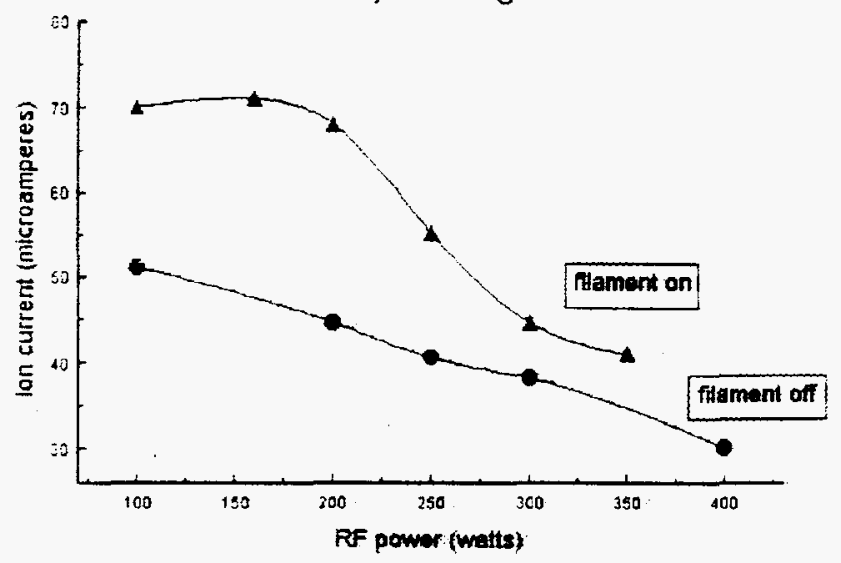

Normalized emittance

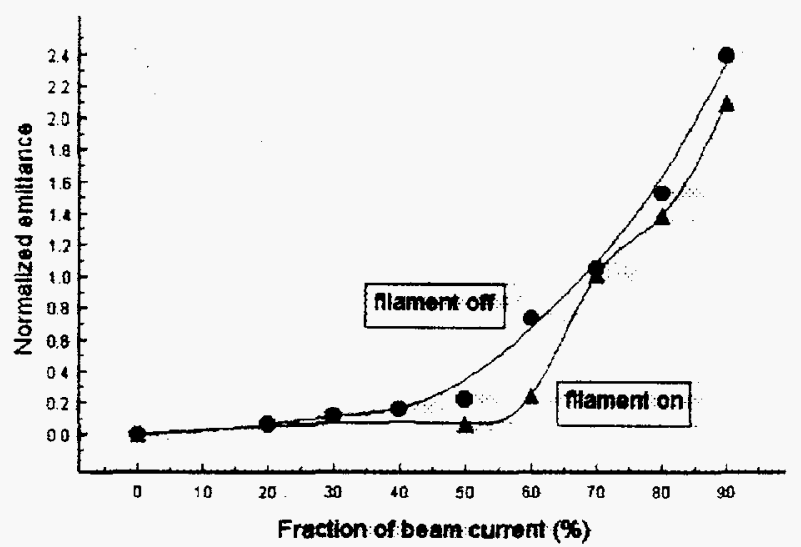

Figure 2: Performance enhancement of the ionization efficiency, beam current, dissociation and emittance achieved through lilament operation. Emittance is given in $\pi \cdot \mathrm{mm} \cdot \mathrm{mrad} \cdot(\mathrm{MeV})^{1 / 2}$

\section{REFERENCES}

1. G.D. Alton, G.D. Mills and J. Dellwo. Rev. Sci. Instrum. 65,2006 (1994)

2. Z.Q. Xie. C.M. Lynies, R.S. Lam and A. Lungdgren, Rev. Sci. Instrum. 62, 775 (1991)

3. T. Nakagawa, T. Kageyama, M. Kase, A. Goto and Y. Yano., Jpn. J. Appl. Phys. 32, L1335 (1993)

4. G. Melin et al. Proc. of the Tenth Int. Workshop on ECR Ion Sources. Knoxville. TN. Nov. 1-2. (1991) p.1

5. T. Nakagawa. T. Kageyama, Jpn. J. Appl. Phys. 30, L1588 (1992)

6. S. Gammino. J. Sijbring and A.G. Drentje, Rev. Sci. Instrum. \$3, 2872 (1992)
7. G. Mouchaty, R.C. Rogers. P. Smelser and D.P. May: Proc. 13th Int. Conf. Cyclotrons \& their Applications, Vancouver (1992) p.369

8. T. Nakagawa, T. Kageyama, M. Kase, A Goto and Y. Yaho Jpn. J. Appl. Phys 31 (1992) L1129

9. Y. Matsubara. H. Tahara. S. Nogawa and J. Ishikawa: Rev. Sci. Instrum. 61 (1990) 541

10. M.E. Abdelaziz. M.M. Abdelbaki and S.G. Zakhary, Rev. Sci. Instrum. 63, 2749 (1992) 


\section{PAC'95 Keyword Selection}

Suggested Keywords - Please select up to 5 keywords \& indicate with a checkmark

\begin{tabular}{|c|c|c|c|c|c|}
\hline Kevword & $\checkmark$ & Kevword & $\checkmark$ & Keyword & $\checkmark$ \\
\hline acceleration & & hadron & & polarization & \\
\hline accelerator-theory & & heavy-ion & D & positron & \\
\hline accumulation & & higher-order-mode & & power-supply & \\
\hline alignment & & impedance & & proton & \\
\hline antiproton & & incoherent-effects & & pulse-stretcher & \\
\hline background & & induction & & pulsed-power & \\
\hline beam-beam-effects & & industrial-accelerators & & quadrupole & \\
\hline beam-cooling & & injection & & radiation & \\
\hline beam-loading & & injector & & radio-frequency & 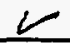 \\
\hline beam-losses & & insertion & & radio-frequency-quadrupole & \\
\hline beam-transport & & insertion-device & & radio-frequency-gun & \\
\hline beat-wave & & instrumentation & & radioactivity & \\
\hline betatron & & interacuion-region & & recirculation & \\
\hline booster & & inverse-free-electron-laser & & resonance & \\
\hline brightness & & ion & 2 & RF-structure & $\bar{U}$ \\
\hline brilliance & & ion-effects & & scattering & \\
\hline bunching & & ion-source & 2 & secondary-beams & \\
\hline cathode & & isotope-production & & separation-scheme & \\
\hline chromatic-effects & & kaon & & septum & \\
\hline civil-engineering & & kicker & & sextupole & \\
\hline closed-orbit & & klystron & & shielding & \\
\hline coherent-effects & & laser & & simulation & \\
\hline collective-effects & & lattice & & single-bunch & \\
\hline collider & & lepton & & site & \\
\hline collimation & & light-ion & & space-charge & \\
\hline controls & & linac & & storage-ring & \\
\hline coupling & & linear-collider & & superconducting-magnet & \\
\hline cryogenics & & linear-dynamics & & superconducting-RF & \\
\hline cyclotron & & longitudinal-dynamics & & superconductivity & \\
\hline damping & & luminosity & & survey & \\
\hline diagnostics & & magnet-design & & synchro-cyclotron & \\
\hline dipole & & man-machine-interface & & synchrotron & \\
\hline dumping & & medical-accelerators & & synchrotron-radiation & \\
\hline dynamic-aperture & & micro-particles & & tandem-accelerator & \\
\hline electromagnetic-fields & & microtroil & & target & \\
\hline electron & & monitoring & & transverse-dynamics & \\
\hline electrostatic-devices & & multi-bunch-effects & & undulator & \\
\hline emituance & & multipole & & vacuum & \\
\hline energy-calibration & & neutral-beams & & Van-der-Graaf & \\
\hline extraction & & non-linear-dynamics & & wakefield & \\
\hline factory & & Octupole & & wiggler & \\
\hline feedback & & operational-performance & & & \\
\hline fibre-optics & & optical-matching & & & \\
\hline focusing & & optics & & & \\
\hline free-electron-laser & & permanent-magnet & & & \\
\hline ground-motion & & photon & & & \\
\hline gun & & pick-up & & & \\
\hline H-minus & & plasma & & & \\
\hline
\end{tabular}

